\title{
ANÁLISIS DEL DISCURSO DE GÉNERO EN LAS MATRICES CURRICULARES DE LAS CIENCIAS DE LA ACTIVIDAD FÍSICA Y EL DEPORTE
}

\author{
GENDER DISCOURSE ANALYSIS IN CURRICULUM MATERIALS OF THE \\ PHYSICAL ACTIVITY AND SPORT SCIENCES SCHOOL
}

\begin{abstract}
ANÁLISE DO DISCURSO DE GÊNERO NAS MATRIZES CURRICULARES SOBRE AS CIÊNCIAS DA ATIVIDADE FÍSICA E DO DESPORTO
\end{abstract}

\author{
Pedrona Serra Payeras*, María Teresa Vizcarra Morales*, \\ Beatriz Garay Ibáñez de Elejalde*, Maria Prat Grau ${ }^{* * *}$, Susanna Soler Prat ${ }^{*}$
}

Sexismo.

Currículum.

Educación superior.

Cambio Social.
Palabras clave:

\begin{abstract}
Resumen: En este trabajo se pretende analizar cómo se ha introducido la perspectiva de género en el currículum universitario, y más específicamente, en el grado de Ciencias de la Actividad Física y el Deporte. Para ello se ha realizado un análisis de contenido y de discurso de 763 guías docentes procedentes de 16 universidades españolas. Los resultados ponen de relevancia como la construcción del conocimiento, y las diversas formas como se puede transmitir conlleva distintas implicaciones para la mayor o menor transformación de las relaciones de género. Las aportaciones de este estudio pueden ser útiles para el diseño de propuestas pedagógicas que pretenden incluir la perspectiva de género y fomentar la transformación social desde la formación universitaria.
\end{abstract}

Keywords: Sexism. Curriculum. Education, Higher. Social change.

Palavras chave: Sexismo.

Currículo.

Educação superior. Mudança Social.
Abstract: This paper aims to analyze how gender perspective has been introduced in the higher education curriculum, specifically in the Physical Activity and Sport Sciences degree. Content analysis and discourse analysis of 763 syllabuses from 16 Spanish public universities were carried out. Findings reveal that construction of knowledge and the several ways it can be conveyed have distinct implications on changing gender relations. The article contributes to the design of new pedagogical proposals that aim to include the gender perspective and promote social changes from higher education.

Resumo: Este trabalho tem como objetivo analisar a introdução da perspectiva de gênero no currículo universitário e, mais especificamente, no grau de Ciências da Atividade Física e do Desporto. Para isso, foi realizada uma análise de conteúdo e discurso de 763 guias de ensino de 16 universidades espanholas. Os resultados mostram como a construção do conhecimento, e as várias maneiras de transmissão trazem implicações diferentes para o grau de transformação das relações de gênero. As contribuições deste estudo podem ser úteis para a concepção de propostas educativas que visam incluir a perspectiva de gênero e promover a transformação social do ensino universitário.
*Instituto Nacional de Educación Física de Cataluña (INEFC-Barcelona). Barcelona, Cataluña, Espanha. E-mail: pserrapayeras@gmail.com

**Universidad del País Vasco. Vitoria, País Vasco. Espanha. E-mail: mariate.bizkarra@ehu.es

***Universidad Autónoma de Barcelona Cerdanyola, Barcelona. Espanha. E-mail: maria.prat@uab.cat

Recebido em: 08-07-2015 Aprovado em: 27-03-2016

(c) (1) (8) Licence 


\section{LA TRANSFORMACIÓN CURRICULAR DESDE LA PERSPECTIVA DE GÉNERO}

La inclusión de la perspectiva de género en los estudios universitarios es un aspecto claramente potenciado desde la legislación universitaria y también deportiva en España (Ley Orgánica 3/2007, para la Igualdad Efectiva entre mujeres y hombres; Ley Orgánica 4/2007, de Universidades - LOU). No obstante, una vez establecidas las políticas universitarias, es necesario analizar en qué medida, y de qué forma, se introduce la perspectiva de género en los materiales curriculares, y más concretamente, en los currículums universitarios. En España, a partir de 2007, son varias las investigaciones que analizan la inclusión de la perspectiva de género en los currículums universitarios, especialmente los vinculados al ámbito educativo (ANGUITA; TORREGO, 2009; ARISTIZABAL; VIZCARRA, 2012; DONOSO; VELASCO, 2013; FLECHA, 1999; HERNÁNDEZ, 2011; PEZZl et al. 2011; TOMÁS, 2012; VENTURA, 2008).

En el ámbito de la Actividad Física (AF) y el deporte, los conocimientos de género que han sido desarrollados desde finales de 1970 hasta nuestros días, constituyen un importante cuerpo teórico (DEVÍS-DEVÍS, 2012). Su inclusión en los estudios superiores se considera fundamental para transformar las relaciones tradicionales de género 'en' y 'mediante' la AF y el deporte, un ámbito caracterizado por su tradición patriarcal y la presencia de actividades fuertemente estereotipadas (DOWLING, 2013; FLINTOFF, 1993; MACDONALD; KIRK; BRAIUKA, 1999; WRIGHT, 2002).

No obstante, los discursos y prácticas patriarcales que subyacen en la cultura profesional dificultan la ruptura del status quo y siguen definiendo lo que se considera como conocimiento valioso para la profesión. Así, el conocimiento sobre género goza de poco espacio en los programas universitarios, un terreno caracterizado por las luchas para definir el conocimiento válido y oficial (ALFARO; VÁZQUEZ, 2007; DEWAR, 1990; DOWLING, 2013; FLINTOFF, 1993, 1998; MACDONALD; KIRK; BRAIUKA, 1999; RICH, 2001; SCRATON, 2013; SILVA et al. 2014; WRIGHT, 2002).Además, más allá de su presencia o no, se debe tener en cuenta que la propia construcción del conocimiento, y la forma como se transmite, puede reproducir las relaciones de género, de modo que es necesario analizar no solo si se incluye o no la perspectiva de género, sino también, cómo está presente. Es decir, cuando es incluida ¿cómo se construye y representa la perspectiva de género? Para realizar este análisis más cualitativo, en este estudio se entiende la perspectiva de género desde una perspectiva amplia, considerando los distintos conceptos y enfoques de las diferentes teorías feministas.

Tal y como sintetizan Scraton y Flintoff (2006), las diferentes corrientes feministas han abordado la distinción entre sexo/género, las relaciones de poder, y el concepto de diferencia de distintas formas. El feminismo liberal se focaliza en la igualdad de oportunidades y de acceso al deporte y la educación física (EF). Sin embargo, una de las críticas de esta corriente es la omisión de la importancia de las relaciones de poder en la sociedad, y como las mujeres quedan subordinas y oprimidas a pesar de los cambios legales. Desde el feminismo estructuralista, en cambio, sí que se hace hincapié en los mecanismos de reproducción de los modelos de masculinidad y feminidad y las resistencias al cambio en las prácticas sociales e institucionales. Finalmente, desde el feminismo post-estructuralista, se plantea la deconstrucción de la visión dicotómica de chico/ chica y de feminidad/masculinidad, y hace hincapié en la multiplicidad de formas de resistencia a los modelos hegemónicos que dispone la persona, de modo que propone cambiar el foco de atención de las limitaciones estructurales a las posibilidades de empoderamiento y resistencia del 
individuo. A pesar de los posicionamientos descritos, estas teorías no son independientes unas de otras sino que se sobreponen (FLINTOFF; SCRATON, 2001).

Desde 1980 han existido las iniciativas de transformación del currículum para promover la inclusión de la perspectiva de género en los estudios superiores en el contexto internacional, especialmente en áreas como la historia, la historia del arte, o la literatura (CHUPPACORNELL, 2005). Si bien son varias las denominaciones utilizadas para referirse a este tipo de transformación curricular: 'estudios de la mujer' o 'women's studies' (CHUPPA-CORNELL, 2005), 'perspectiva feminista' (MCINTOSCH, 1984), 'estudios de género' (VENTURA, 2008), 'gender mainstreaming' (MORLEY, 2007). En las últimas décadas, se han desarrollado varias escalas, o Phase Theories, para evaluar las diferentes formas de inclusión de los conocimientos sobre las mujeres y las relaciones de género. Entre ellas, destaca la iniciada por Tetreault (1986), que ha sido utilizada en sucesivos trabajos (GRUNIG, 2006; MAHER; TETREAULT, 2011).

Tetreault (1986) desarrolló su propuesta con 5 fases o niveles de inclusión: 1. Conocimiento masculino; 2. Conocimiento compensatorio; 3. Conocimiento bifocal; 4. Conocimiento feminista; y 5. Conocimiento multifocal o relacional. Chuppa-Cornell (2005) describe las características de la propuesta de Tetreault: en la primera fase se considera la experiencia masculina como la experiencia universal, con un carácter claramente androcéntrico. En la segunda fase, al advertirse el olvido o la invisibilidad de las mujeres, se incorporan algunos nombres de mujeres, si bien este planteamiento refuerza la idea que la mayoría de las mujeres son irrelevantes o no merecen atención excepto algunas excepciones. El planteamiento bifocal, en la fase 3, presenta la experiencia humana bajo la perspectiva dicotómica del mundo femenino y el mundo masculino, destacando especialmente las diferencias entre hombres y mujeres. En este caso, se corre el peligro de devaluar la esfera femenina, de poner demasiado énfasis en la pasividad femenina y en la victimización de las mujeres, además de pasar por alto las importantes diferencias en el grupo de mujeres. La fase 4 pone el centro de atención en las mujeres y en sus vidas, y tiene en cuenta la intersección de otras categorías sociales como la clase social, la raza, la orientación sexual, etc. Así, recoge aportaciones de múltiples disciplinas y las múltiples facetas que pueden tener las mujeres. Finalmente, la fase 5 es aquella en que se sigue la tarea de la fase anterior e incorpora también aspectos vinculados a las experiencias de los hombres, con un enfoque pluralístico y holístico que busca una visión conjunta de las experiencias masculina y femenina superando la división dicotómica de fases anteriores.

Junto a la escala de Tetreault (1986) se puede destacar el trabajo de McIntosh (1984), basado en estudios de Historia. Añade el carácter interactivo existente entre las diferentes fases, remarcando que todas las fases se pueden dar al mismo tiempo en una disciplina. Estas escalas se han utilizado para valorar el nivel de transformación alcanzado en libros de texto, medios de comunicación, artículos científicos, o currículums universitarios (CHUPPACORNELL, 2005).

Otro aspecto a considerar es la relevancia del análisis de los materiales curriculares, que entendidos de forma amplia, incluyen recursos que se utilizan en la planificación, el desarrollo y la evaluación de la enseñanza (DEVÍS-DEVÍS et al. 2001). En el caso universitario, las guías docentes son el material en el que el profesorado da a conocer su asignatura, tienen que ser públicas, y están sujetas a las directrices legales. Por ello, el discurso presente en este material es una poderosa herramienta que contribuye a la construcción del conocimiento (SVENDSEN; SVENDSEN, 2014). 
A partir de este marco político, teórico y académico se aborda el discurso de género en el currículum del grado universitario de Ciencias de la Actividad Física y el Deporte (CAFyD) a través del análisis de una muestra de guías docentes de universidades públicas españolas.

\section{MÉTODO}

Se han analizado 763 guías docentes de 16 universidades públicas, de un total de 37 (públicas y privadas), que impartían el grado de CAFyD durante el curso 2012/2013. Mediante un muestreo intencional (PATTON, 1990), y considerando la mayor relevancia del sistema universitario público en España en cuanto a tradición científica, número de estudiantes y centros (ESPAÑA, 2012), se han seleccionado las universidades públicas de cada comunidad autónoma en que se ofrecen estos estudios y que tuvieran los cuatro cursos del grado implantados. También era imprescindible que hubiera acceso a las guías docentes mediante la página web. Se observó que en aquellas universidades con mayor oferta de optatividad, el número de asignaturas era muy superior, si bien en algunos casos, no en todas las asignaturas estaban disponibles sus guías.

Como Silva, Botelho y Queirós (2006), la realización del estudio implicó dos fases en el proceso de análisis. En una primera fase del estudio se realizó un análisis descriptivo y cuantitativo del nivel de visibilización de la perspectiva de género en todas las guías docentes y de su coherencia desde una perspectiva pedagógica en los diferentes apartados que debe contener la guía según ANECA (2012): denominación de la asignatura, tipología (obligatoria u optativa), competencias, contenidos, objetivos, actividades formativas, evaluación y bibliografía. Se analizó la utilización de los siguientes términos: coeducación, mixto/a, discriminación, equidad, igualdad, feminismo, género, sexo, hombre(s), y mujer(es) (ver Cuadro 1).

Cuadro 1 - Universidades de la muestra y número de guías docentes

\begin{tabular}{lc}
\hline Universidad & № guías \\
\hline U. A Coruña & 44 \\
U. Politécnica de Madrid & 112 \\
U. Las Palmas de Gran Canaria & 42 \\
U. de Zaragoza & 44 \\
U. de Lleida & 42 \\
U. de Murcia & 39 \\
U. de Barcelona & 51 \\
U. Castilla la Mancha & 43 \\
U. de Vigo & 41 \\
U. Alcalá de Henares & 32 \\
U. de León & 73 \\
U. de Extremadura & 37 \\
U. del País Vasco & 26 \\
U. Autónoma de Madrid & 51 \\
U. de Sevilla & 50 \\
U. de Granada & 36 \\
\hline TOTAL & 763 \\
\hline
\end{tabular}


En una segunda fase se realizó un análisis del discurso de las 54 asignaturas que visualizan la perspectiva de género. El análisis del discurso explora cómo el lenguaje contribuye a reproducir las relaciones de poder y permite realizar diferentes lecturas analíticas de los textos (VAN DIJK, 2009). Del mismo modo que Rossi et al. (2009), se ha considerado las guías docentes como un texto que se configura a partir de múltiples discursos. El discurso que se desprende del tipo de lenguaje utilizado, lo que se dice y no se dice, como señala Sparkes (1990), articula y refleja las ideas, creencias, valores y prácticas de quién lo emite.

A partir de los datos obtenidos en las dos fases de análisis se ha establecido una categorización de las diferentes formas de abordar la perspectiva de género a partir de un proceso inductivo-deductivo que ha permitido clasificar el conjunto de asignaturas. Deductivo, porque parte de la revisión de la literatura realizada sobre la perspectiva de género en AF y deporte; e inductivo, porque se ha conformado, durante el propio proceso de análisis. Las cinco categorías establecidas son: 1 . Ausencia de los conocimientos sobre las mujeres y su experiencia; 2. Presencia superficial o 'políticamente correcta'; 3. Reproduciendo a mujeres (débiles, pasivas y oprimidas), y a hombres; 4. Reconociendo lo femenino y las múltiples diferencias; y, 5. Transformación de las relaciones de género.

Las asignaturas analizadas quedaron distribuidas en cada una de las categorías tal y como aparece en cuadro 2.

Cuadro 2 - Guías recogidas en cada una de las categorías

\begin{tabular}{lcc}
\hline Categoría de análisis & Frecuencia & $\%$ \\
\hline 1. Ausencia de los conocimientos sobre las mujeres y su experiencia & 569 & 74.6 \\
2. Presencia superficial o "políticamente correcta" & 141 & 18.4 \\
3. Reproduciendo a mujeres (débiles, pasivas y oprimidas), y a hombres & 27 & 3.5 \\
4. Reconociendo lo femenino y las múltiples diferencias & 20 & 2.6 \\
5. Transformación de las relaciones de género & 7 & 0.9 \\
\hline TOTAL & 763 & $100 \%$ \\
\hline
\end{tabular}

Fuente: Elaboración propia

\section{RESULTADOS Y DISCUSIÓN}

\subsection{Ausencia de los conocimientos sobre las mujeres y su experiencia}

Análisis de las fichas docentes ha permitido observar que en el $74.6 \%$ de asignaturas no hay mención alguna a términos que puedan hacer referencia a la perspectiva de género. Desde las diversas áreas de conocimiento que se abordan en los estudios de CAFyD (salud, educación, gestión, recreación, o entrenamiento deportivo), destaca la ausencia de referencias a la experiencia y realidad de las mujeres 0 a la influencia de los modelos y relaciones de género en la práctica deportiva.

Tal y como apuntan los trabajos de Dowling (2013), Wright (2002), Macdonald, Kirk y Braiuka (1999), Flintoff (1993) y Dewar (1990) la incorporación de la perspectiva de género en el campo de la AF y el deporte resulta difícil y controvertida. 
Sobresale especialmente la ausencia del término 'coeducación' en las asignaturas de pedagogía o didáctica, tal y como recoge también Valdivia-Moral et al. (2012). Si bien históricamente la EF ha sido un área escolar con una clara carga ideológica en cuanto a las relaciones de género, parece que hoy este enfoque coeducativo no es trascendente. La falta de formación en coeducación, y el desconocimiento sobre su significado, refuerza la idea de que la implantación de la educación mixta implica la igualdad entre chicos y chicas, tal y como planteaba el feminismo liberal. No obstante, como en el resto de materias escolares (SUBIRATS; BRULLET, 1988), la aparente igualdad que suponía la EF mixta, comportó la anulación de la diferencia, e implicó la implantación de un modelo de EF androcéntrico en el que las actividades próximas a los intereses y experiencias de las niñas son ignoradas 0 menospreciadas, tal y como han denunciado las corrientes feministas posteriores. Abordar el significado de coeducar desde una perspectiva crítica y estimular la reflexión sobre las propias creencias entre el alumnado es necesario para formar sujetos autónomos y emancipados, tal y como proponen Betti et al. (2014). Sin embargo, no parece un concepto oportuno para una parte importante del profesorado.

Destaca también la ausencia del término 'género' en la asignatura de 'Sociología de la actividad física y el deporte', siendo una categoría básica en esta disciplina. Si bien aparecen otras categorías (edad, cultura, nivel socio-económico, etc.), la perspectiva de género se diluye bajo la denominación 'aspectos socioculturales'. Esta omisión apunta a la 'ceguera en materia de género' por parte del profesorado universitario señalada por Donoso y Velasco (2013), el 'espejismo de la igualdad' (VALCÁRCEL, 2008), o bien al 'miedo al feminismo' (WEINER, 2000).

\subsection{Presencia superficial o 'políticamente correcta'}

En un $18.4 \%$ de asignaturas se incluye alguna competencia establecida en el plan de estudios relativa a la perspectiva de género, aunque en ningún otro apartado se encuentra otra alusión. Así se ajustan a la legislación establecida, pero se intuye que sin mucha convicción ya que no desarrollan estas competencias ni en forma de resultados de aprendizaje, contenidos, en metodología, o en la bibliografía. Coincidiendo con Pezzi et al. (2011), se incluye la perspectiva de género en las competencias generales del título, pero éstas no se desarrollan en las guías docentes, realizando un discurso 'políticamente correcto'.

La poca concreción de las nuevas políticas de género, y la falta de una guía para su puesta en práctica, llevan a que no se materialice en las fichas docentes, tal como denuncian Anguita y Torrego (2009). Las leyes de igualdad efectiva han llevado a una corrección formal en muchos documentos oficiales pero posteriormente no se mantiene en la parte específica de la asignatura, redactada por el profesorado.

El desarrollo de la política curricular no implica necesariamente que se aplique, siendo necesario analizar cómo el profesorado aplica esta política, tal y como proponen Soares y Fonseca (2012). Desde la óptica del feminismo liberal podría parecer que esta inclusión es un importante paso adelante y un logro de la legislación vigente. Sin embargo, si no hay un desarrollo que refleje su incorporación en los diferentes aspectos didácticos de las asignaturas, cabe preguntarse sobre los efectos reales de estas medidas más bien formales. Unas medidas formales que, si bien pueden servir de 'escudo' ante los requerimientos legales, pueden convertirse en una herramienta para eludir cambios de contenido más profundos (ANGUITA; TORREGO, 2009). 


\subsection{Reproduciendo a mujeres y a hombres}

Los elementos presentes en esta categoría implican la inclusión de conocimientos relacionados con la mujer, pero la forma de presentarse puede inducir a reproducir los modelos tradicionales de género. Hablar de las cuestiones que afectan a las mujeres puede parecer un avance respecto al 'olvido' que se indica en las categorías anteriores, pero puede ser aún más problemático si refuerza la visión dicotómica entre lo femenino y lo masculino, tal y como denuncia el feminismo post-estructuralista.

Es curioso observar que cuando aparece el conocimiento sobre mujeres es para abordar su función reproductiva, con temas como 'Actividad física durante el embarazo' o 'Actividades acuáticas para embarazadas', que son contenidos encontrados en asignaturas vinculadas a las actividades acuáticas, a poblaciones con necesidades específicas, fisiología del ejercicio, anatomía o entrenamiento deportivo.

En algunos casos también se incluyen referencias a los aspectos biológicos y fisiológicos propios de las mujeres. No obstante, este contenido suele aparecer clasificado como 'otros', 'consideraciones especiales' o 'diferencias sexuales', que suelen estar al final del programa, en un bloque aparte, situando los asuntos de la mujer como una cuestión menor. El modelo es el cuerpo masculino - joven y blanco -.

El sujeto objeto de atención en estas asignaturas es básicamente el hombre, y cuando aparece la mujer es para subrayar las diferencias en sus capacidades de desarrollo motriz, asumiendo el colectivo de mujeres como un grupo homogéneo en el que se ignoran las diferencias intrasexo. Las mujeres se hacen presentes sin tener en cuenta la propia heterogeneidad existente dentro del colectivo en función del nivel de práctica, el biotipo, la clase social, etc., acentuando, así, la construcción dicotómica de los cuerpos de hombres y mujeres conllevando a la reproducción de modelos de género hegemónicos (PFISTER, 2010).

Por otra parte, desde la sociología y la pedagogía, si solo mencionan las estadísticas de participación o la falta de interés de las niñas, se puede generar un discurso donde las mujeres son el 'problema', pues participan menos y no están motivadas para la práctica deportiva. Como señala el feminismo estructuralista, es necesario reconocer las diferencias que se construyen socialmente y las relaciones de poder existentes en las interacciones sociales y en las organizaciones deportivas para cuestionar la hegemonía del modelo androcéntrico. Sin embargo, para evitar una visión propia del determinismo sociocultural, sería necesario también indagar sobre las diversas masculinidades y feminidades, así como mostrar ejemplos de cambio de los modelos tradicionales y de empoderamiento de las mujeres.

En los ámbitos relacionados con la biología y la salud en los estudios de CAFyD, se observa el sesgo de género identificado también en el ámbito médico (ROSSER, 1997). A menudo los datos y saberes transmitidos se corresponden con la población masculina, de modo que el conocimiento se transmite como si fuera válido para ambos sexos. En relación con este aspecto, Hartman-Tews (2011), señala que en la medicina del deporte o la teoría del entrenamiento, el sesgo de género se puede dar por omisión de las diferencias o por la sobredimensión de las diferencias. No obstante, parece que la mujer embarazada sí existe. En relación con esta cuestión, Goellner (2008) destaca que muchos discursos solo tienen en 
cuenta la feminidad que adopta la maternidad, de modo que se considera impropia cualquier actividad que ponga en peligro esta función.

La inclusión de la perspectiva de género bajo una perspectiva dicotómica, jerarquizada y determinística, ya sea por características biológicas o sociales, sigue reforzando los modelos tradicionales, naturalizando y normalizando las diferencias y desigualdades, en lugar de desafiarlas y transformarlas.

\subsection{Reconociendo lo femenino y las múltiples diferencias}

Los elementos que caracterizan esta categoría tienen relación con el reconocimiento de las aportaciones y las experiencias de las mujeres y dan valor a la cultura deportiva femenina, visualizando la diversidad existente entre las diferentes mujeres, así como entre los hombres. Se presentan a las niñas, jóvenes y mujeres como personas con intereses y gustos diferentes, no se describe sólo como un colectivo oprimido, sino como un grupo que participa y aporta a la sociedad y enriquece la cultura deportiva con sus formas de vivir su cuerpo y la AF.

El reconocimiento de la(s) cultura(s) deportiva(s) femenina(s) implica examinar la situación de desigualdad existente entre hombres y mujeres, pero destacando también la menor presencia de los hombres en aquellas actividades consideradas tradicionalmente como femeninas.

Desde un enfoque más cercano al post-estructuralismo, el concepto de igualdad se refiere a igualdad en derechos y deberes respetando las diferencias, y eso no significa hacer lo mismo tomando de referencia el modelo masculino. Se incide en las limitaciones del concepto de igualdad de oportunidades y se pone el acento en la igualdad efectiva.

Otro aspecto que contribuye al reconocimiento de la diferencia y la ruptura de la visión dicotómica de hombres y mujeres es cuando se aborda la construcción cultural del cuerpo con el análisis, por ejemplo, de los 'Significados del cuerpo'. Uno de los elementos que podría favorecer la ruptura de esta dicotomía es abordar la realidad de las personas intersexuales, transgénero o transexuales.

Cuando se aborda la promoción de la salud no solo como ausencia de enfermedad sino como estado de bienestar físico, psicológico y social, se tienen en cuenta los aspectos psicosociales, que dan cabida a comprender que los modelos y relaciones tradicionales de género influyen en la propia concepción de salud y enfermedad. Se reconoce el sesgo existente en los estudios relacionados con la salud y en la medicina deportiva, introduciendo conocimientos vinculados a la salud y al ejercicio de las mujeres que van más allá de los aspectos ginecológicos y de reproducción, como 'la triada de la deportista' tratada como una patología más, sin ser catalogada como 'otras'.

\subsection{Trasformación de las relaciones de género}

El análisis realizado ha permitido también recoger enfoques que apoyan la transformación de las relaciones de género en y mediante la AF y el deporte. Como propone Flecha (1999), en algunas asignaturas se manifiesta el compromiso con la práctica y la transformación social, yendo más allá del discurso teórico, sustituyendo el dualismo mente-cuerpo por la atención a 
la complejidad de la persona, incluyendo también la realidad de los hombres en la perspectiva de género. Se plantea una visión crítica de la construcción del conocimiento y los sesgos androcéntricos, y se proponen metodologías que fomenten la reflexión entre el alumnado sobre sus identidades de género y el impacto que tiene en la sociedad.

Los elementos que caracterizan las asignaturas en esta categoría son similares a los de la categoría anterior, en la línea del feminismo post-estructuralista, ya que se reconocen las diferencias, pero en este caso, se muestra un compromiso explícito con la promoción de la igualdad efectiva entre hombres y mujeres, y una voluntad de mejorar y transformar la realidad reconociendo las múltiples identidades existentes. Encajando con el feminismo postestructuralista, se plantea el género como una categoría relacional, considerando el resto de características socioculturales, tales como, el origen cultural, el nivel socio-económico, la religión, la tendencia sexual, la edad o la discapacidad.

Se observa una clara intención de visibilizar las situaciones de desigualdad y discriminación por razón de género. Se aportan herramientas para la transformación y ejemplos de buenas prácticas que permiten promover el empoderamiento de las mujeres. Así, uno de sus rasgos definitorios es el interés para que el alumnado pueda pasar a la acción. Por ejemplo, la asignatura optativa 'Innovación y cambio sociocultural en la AF y el deporte', de la Universidad de Barcelona, incorpora el compromiso de transformación en el propio título de la asignatura, en los objetivos, en la 'realización de propuestas innovadoras para su transformación', así como la incorporación de 'experiencias en el ámbito de la AF y el deporte que favorecen la participación de los colectivos minoritarios'. La asignatura de 'Sociología de la AF y el deporte' de la Universidad de Coruña (UDC), se propone 'intervenir para modificar los modelos tradicionales de género', y en la asignatura 'Planificación y organización de sistemas y actividades deportivas', de la Universidad de Granada, se plantea como objetivo 'Desarrollar un plan de igualdad en un sistema deportivo'.

Se aborda también la cuestión de la masculinidad hegemónica o las masculinidades, dando un enfoque holístico a la perspectiva de género, de modo que no solo afecta a las mujeres, sino al conjunto de la sociedad, hombres incluidos. En la asignatura optativa 'Mujer y deporte', a pesar de que por su título podría parecer orientada únicamente a la cuestión femenina, entre sus contenidos se aborda la cuestión de la masculinidad: 'Género y deporte: masculinidad y feminidad'; '¿Educación física: un lugar de masculinidades?’.

Se promueve la comprensión de los procesos de reproducción social y cultural en la sociedad, y fomentan la capacidad crítica del alumnado ante los fenómenos de su entorno. Una característica habitual en estos enfoques es estimular la reflexión sobre las propias creencias y revisar su identidad profesional. En la asignatura 'Teoría e Historia' de UDC, una de las tareas de evaluación es la realización de un 'trabajo sobre la propia historia personal', y desde 'Deporte y Educación', de la Universidad Autónoma de Madrid, se da gran importancia a que 'los estudiantes sean capaces de analizar críticamente los estereotipos y tópicos sobre el deporte', o a la 'Elaboración de propuestas de acción positiva', desde la asignatura 'Educación física y diversidad: análisis y propuestas', de la misma universidad.

Desde la promoción de la salud, además de contemplar la influencia del entorno y los estereotipos de género en los hábitos de salud y el concepto de enfermedad, se proponen también medidas de salud colectiva que generen entornos saludables que rompan con el dualismo cartesiano mente-cuerpo y la presión social por encajar en los cánones de feminidad 
y masculinidad hegemónicas. Se da una visión crítica de la 'sociedad de consumo del cuerpo', y de cómo los estereotipos presentes en los medios de comunicación, publicidad, moda, e incluso en el deporte pueden promover hábitos no saludables, generar una baja autoestima, y provocar trastornos de conducta alimentaria.

\section{COMENTARIOS FINALES}

Tal y como sucede en otros campos científicos y académicos (CHUPPA-CONNELL, 2005; MCINTOSH, 1984; GRUNIG, 2006; MAHER; TETREAULT, 2011), este estudio constata que el conocimiento relacionado con la perspectiva de género que se incluye en la formación en CAFyD puede presentarse de varias formas, con distintas implicaciones para la transformación de las relaciones de género, coincidiendo con los diversos prismas de las diferentes corrientes feministas.

En conjunto, este trabajo no sólo nos permite conocer los discursos existentes en las guías docentes universitarias de CAFyD, sino también identificar el potencial transformador que existe detrás de cada asignatura. Con ello esperamos que pueda servir como elemento de reflexión para aquellas personas que se dedican a la enseñanza universitaria.

Como limitaciones de este estudio, cabe señalar la necesidad de analizar también la situación en las universidades privadas en las que, por su juventud y su interés por adaptarse a la demanda e intereses del alumnado en su oferta formativa, tal y como describe Karhus (2012), pueden encontrarse circunstancias distintas a las descritas. Por otra parte, se debe señalar que por el hecho de que en las guías docentes aparezcan términos vinculados a la perspectiva de género, no implica que se introduzca en la docencia en el aula, y del mismo modo, que no se mencione, no implica que no se introduzca. Por ello, se considera relevante observar si se visibiliza, o no, la perspectiva de género en estos documentos ya que son los que están sujetos a la legalidad universitaria y son los que dan a conocer los conocimientos al alumnado y a la comunidad científica. Además, se considera conveniente seguir este estudio analizando el resto de materiales curriculares que se utilizan así como la praxis en el aula.

\section{REFERENCIAS}

AGENCIA ANDALUZA DEL CONOCIMIENTO. Guía de apoyo para la elaboración de la memoria de verificación de títulos oficiales universitarios. Madrid: ANECA, 2012. Disponible en: <http://deva.aac.es/include/files/universidades/verificacion/02-Guia de apoyo VERIFICACION AAC V02 111018.pdf>. Acceso en: 25 feb. 2016.

ALFARO, Élida; VÁZQUEZ, Benilde. La formación con perspectiva de género en los estudios de Ciencias de la Actividad Física y el Deporte. In: ACTAS DEL SEMINARIO PERMANENTE MUJER Y DEPORTE. Madrid: INEF - Universidad Politécnica de Madrid, 2007. p. 1-7.

ANGUITA, Rocío; TORREGO, Luís. Género y Educación. Revista Interuniversitaria de Formación del Profesorado, Zaragoza, v. 64, n. 23, p. 17-25, 2009.

ARISTIZABAL, Pilar; VIZCARRA, Maria Teresa. Illustration of gender stereotypes in the initial stages of teacher training education. Education, London, v. 2, n. 7, p. 347-355, Jun. 2012.

BETTI, Mauro et al. Fundamentos filosóficos e antropológicos da Teoria do Se-movimentar e a formação de sujeitos emancipados, autônomos e críticos: o exemplo do currículo de Educação

Física do Estado de São Paulo. Movimento, Porto Alegre, v. 20, n. 4, p. 1631-1653, out./dez. 2014. 
CHUPPA-CORNELL, Kim. The conditions of difficulty and struggle?: A discovered theme of curriculum transformation and women's studies discourse. NWSA Journal, Baltimore, v. 1, n. 17, p. 23-24, 2005.

DEVÍS-DEVÍS, José et al. Los materiales curriculares impresos en educación física: clasificación, usos e investigación. Movimento, Porto Alegre, v. 7, n. 15, p. 119-136, 2001.

DEVÍS-DEVÍS, José. La investigación socio crítica en la educación física. Estudios Pedagógicos, Valdivia, v. 38, n. especial, p. 125-153, 2012.

DEWAR, Alison. Oppression and privilege in physical education: struggles in the negotiation of gender in a university programme. In: KIRK, David; TINNING, Richard. Physical education, curriculum and culture. Londres: Falmer, 1990. p. 67-99.

DONOSO, Trinidad; VELASCO, Anna. ¿Por qué una propuesta de formación en perspectiva de género en el ámbito universitario? Profesorado: Revista de Curriculum y Formación del Profesorado, Granada, v. 17, n. 1, p. 71-88, 2013.

DOWLING, Fiona. Teacher educator's gendered worplace tales. In: PFISTER, Gertrud; SISJORD, Mari Kristin. Gender and sport: changesand challenges. Münster: Waxmann, 2013. p. 217-231.

ESPAÑA. Ley Orgánica 3/2007, de 22 de marzo, para la igualdad efectiva de mujeres y hombres.

Boletín Oficial del Estado, n. 71, p. 12611-12645, 12 jun. 2014.

ESPAÑA. Ley Orgánica 4/2007, de 12 de abril 2007, por la que se modifica la Ley Orgánica 6/2001, de 21 de diciembre, de Universidades. Boletín Oficial del Estado, n. 89, p. 1624116260, 13 abr. 2007.

ESPAÑA. Ministerio de Educación Cultura y Deporte. Datos y cifras del sistema universitario español. Madrid. Disponible en: <http://www.mecd.gob.es/dms/mecd/educacion-mecd/areaseducacion/universidades/estadisticas-informes/estadisticas-informes-documentum/datos-cifras/20122013-datos-y-cifras-sistema-universitario-espanol.pdf>. Acceso en: 10 feb. 2015.

FLECHA, Consuelo. Género y ciencia. A propósito de los "Estudios de la mujer" en las universidades. Educación XX1, Madrid, v. 2, p. 223-244, 1999.

FLINTOFF, Anne. Gender, physical education and initial teacher education. In: EVANS, John. Equality, education and physical education. Londres: Falmer, 1993. p. 184-204.

FLINTOFF, Anne. Sexism and homophobia in physical education: The challenge for teachers educators. In: GREEN, Ken; HARDMAN, Ken. Physical education: a reader. Aachen: Meyer \& Meyer, 1998. p. 291-313.

FLINTOFF, Anne; SCRATON, Sheila. Stepping into active leisure? Young women's perceptions of active lifestyles and their experiences of school physical education. Sport, Education and Society, London, v. 6, n. 1, p. 5-21, 2001.

GOELLNER, Silvana Vilodre. Deporte y cultura fitness: la generización de los cuerpos contemporáneos. Revista Digital Universitaria, México, v. 9, n. 7, 2008. Disponible en: <http:// www.revista.unam.mx/vol.9/num7/art47/art47.htm>. Acceso en: 10 feb. 2015.

GRUNIG, Larissa A. Feminist phase analysis in public relations: where have we been? Where do we need to be? Journal of Public Relations Research, Hamdem, v. 2, n. 18, p. 115-140, Feb. 2006.

HARTMANN-TEWS, Ilse. Gender bias in research - a case study in sports medicine. In: Dones i esport: bones pràctiques en recerca, docència i innovació. Barcelona: INEFC, 2011. Disponible en: https://inefcgiseafe.files.wordpress.com/2011/09/prof-dr-hartmann-tews-ilse-dr-rulofs-bettina-profapl-dr-brixius-klara-dr-claudia-combrink gender-bias-in-research-e28093-a-case-study-in-sportsmedicine.pdf. Acceso en: 29 oct. 2015. 
HERNÁNDEZ, Ana María. Una nueva mirada a la formación docente como camino hacia equidad de género. Revista Electrónica Educare, Heredia v. 15, n. 1, p.123-134, 2011. Disponible en: <http://www.revistas.una.ac.cr/index.php/EDUCARE/article/view/885>. Acceso en: 19 feb. 2015.

MACDONALD, Doune; KIRK, David; BRAIUKA, Sandy. The social construction of the physical activity field at the school/university interface. European Physical Education Review, Londres, v. 1, n. 5, p. 31-52, Feb. 1999.

MAHER, Frances A; TETREAULT, Mary Kay. Long-term transformations: excavating privilege and diversity in the academy. Gender and Education, London, v. 3, n. 23, p. 281-297, May 2011.

MCINTOSH, Peggy. Interactive phases of curricular re-vision: a feminist perspective. In: SPANIER, Boniee; BLOOM, Alexander; BOROVIAK, Darlene. Toward a balanced curriculum: a source book for initiating gender integration projects. Chicago: Schenkman, 1984.

MORLEY, L. Sister-matic: gender mainstreaming in higher education. Teaching in Higher Education, London, v. 5-6, n. 12, p. 607-620, 2007.

PATTON, Michael Quinn. Qualitative evaluation and research methods. Newbury Park: Sage, 1990.

PEZZI, Pilar et al. La formación con perspectiva de género en los estudios de Ciencias de la Actividad Física y el Deporte. In: CONGRESO INTERNACIONAL DE EDUCACIÓN PARA LA IGUALDAD: GÉNERO Y SEXUALIDADES. Granada: Natívola, 2011. p. 310-319.

PFISTER, Gertrud. Women in sport: gender relations and future perspectives. Sport in Society, London, v.13, n. 2, p. 234-248, Feb. 2010.

$\mathrm{RICH}$, Emma. Gender positioning in teacher education in England: New rhetoric, old realities. International Studies in Sociology of Education, London, v. 11, n. 2, p. 131-156, 2001.

ROSSER, Sue. V. Health: discipline analysis. Baltimore: National Center for Curriculum Transformation Resources on Women, 1997. (Women in the curriculum series).

ROSSI, Tony et al. With the best of intentions: a critical discourse analysis of physical education curriculum materials. Journal of Teaching in Physical Education, Champaign, v. 1, n. 28, p. 75-89, 2009.

SCRATON, Sheila. Feminism and physical education: does gender still matter? En: PFISTER, Gertrud; SISJORD, Mari Kristin. Gender and sport. Changes and challenges. Münster: Waxmann, 2013. p. 199-216.

SCRATON, Sheila; FLINTOFF, Anne. Girls and PE. In: KIRK, D; O'SULLIVAN, M; MACDONALD, D. Handbook of research on physical education. Londres: Routledge, 2006. p. 796-783.

SILVA, Ana Márcia; SOLER, Susanna; PUIG, Núria; PRAT, Maria. El proceso Bolonia y sus efectos en el campo de las ciencias de la actividad física y del deporte en España. Movimento, Porto Alegre, v. 20, n. 3, p. 1061-1082, jul./set. 2014.

SILVA, Paula; BOTELHO GOMES, Paula; QUEIRÓS, Paula. Educação física, desporto e género: o camino percorrido na Faculdade de Desporto da Universidade do Porto (Portugal). Movimento, Porto Alegre, v. 12, n. 1, p. 31-58, jan./abr. 2006.

SOARES JÚNIOR, Neri Emilio.; FONSECA BORGES, Lívia Freitas. A pesquisa na formação inicial dos professores de educação física. Movimento, Porto Alegre, v. 18, n. 2, p. 169-186, abr./ jun. 2012.

SPARKES, Andew. Curriculum change and physical education: towards a micropolitical understanding. Geelong, Australia: Deakin University, 1990. 
SUBIRATS, Marina; BRULLET, Cristina. Rosa y azul: la transmisión de los géneros en la escuela mixta. Madrid: Ministerio de Cultura, 1988.

SVENDSEN, Annemari Munk; SVENDSEN, Jesper Tinggaard. Teacher or coach? How logics from the field of sports contribute to the construction of knowledge in physical education teacher education pedagogical discourse through educational texts. Sport, Education and Society, Champaing, sept. 2014. Disponible en: http://www.tandfonline.com/doi/ abs/10.1080/13573322.2014.956713?journalCode=cses20. Acceso en: 13 ene. 2016.

TETREAULT, Mary Kay. Integrating Women's History: the case of United States history high school text books. The History Teacher, New York, v. 19, n. 2, p. 211-261, Feb. 1986.

TOMÁS, Marina. Conflictos en las universidades por razones de planes de estudios nuevos. In: DE LA HERRÁN, Agustín; PAREDES, Joaquín. Promover el cambio pedagógico en la universidad. Madrid: Pirámide, 2012. p. 151-168.

VALCÁRCEL, Amelia. Feminismo en el mundo global. Madrid: Cátedra, 2008.

VALDIVIA-MORAL, Pedro Angel; LÓPEZ-LÓPEZ, Miriam; LARA-SÁNCHEZ, Amador Jesús; ZAGALAZ-SÁNCHEZ, Maria Luisa. Concepto de coeducación en el profesorado de Educación Física y metodología utilizada para su trabajo. Movimento, Porto Alegre, v. 18, n. 4, p. 197-217, out. /dez. 2012.

VAN DIJK, Teun A. Society and discourse: how social contexts influence text and talk. Cambridge: Cambridge University, 2009.

VENTURA, Asunción. Normativa sobre estudios de género y universidad. Feminismo/s, Alicante, n. 12, p. 155-184, dic. 2008.

WEINER, Gaby. A critical review of gender and teacher education in Europe. Pedagogy, Culture \& Society, London, v. 8, n. 2, p. 233-247, July 2000.

WRIGHT, Jan. Physical education teacher education: sites of progress or resistance. In: PENNEY, Dawn. Gender and physical education: contemporary issues and future directions. Londres: Routledge, 2002. p. 190-207. 
Apoyo Financiero: Este trabajo forma parte del proyecto "La Igualdad en Juego: la (no)presencia de las mujeres y la perspectiva de género en los estudios de la familia de la Actividad Física y el Deporte" (DEP2012-31275), subvencionado por el Ministerio. 